the New Physics has been crucial in strengthening our confidence. But can the News Physics help us to solve the tantalizing difficulties of the paradoxical quark behaviour?

Many physicists have expressed strong hopes that the spectrum and transition rates of the $\mathrm{J}-\Psi$ family, the "Charmonium" system as it has been called (paraphrasing the "positronium") can be to the finding of the correct quark theory, what the hydrogen atom has been to the formulation of Quantum Mechanics. Another lesson we have learned is that, through the quark concept, the weak interactions exhibit a remarkable symmetry between leptons and hadrons. It will be very interesting to see whether this symmetry survives the possible discovery of new types of leptons and/or hadrons (some peculiar events found at SPEAR indicate that a heavy lepton has already been found).

It is really within high energy weak interactions, with their quark-lepton symmetry, that matter could one day show truly universal features, thus leading to a unification of all forms of interactions. Such grandiose schemes are presently being conceived, a legitimate offspring of the New Physics.

Who knows; is the J- $\Psi$ the first link of a chain leading to the fulfilment of the ancient aspiration to cosmic unity?

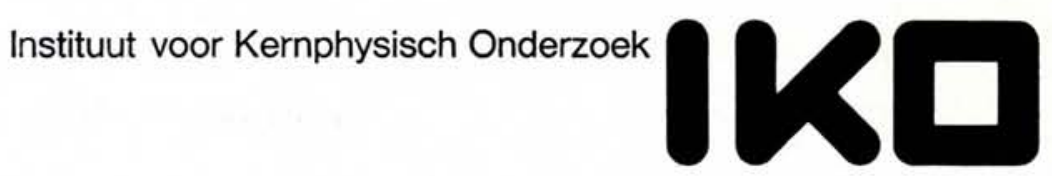

Applications are invited for the position of :

\section{Groupleader}

\section{in Pion-Muon Nuclear Physics}

at the Institute for Nuclear Physics Research (IKO), Amsterdam.

The Institute is in the course of constructing a high duty cycle $500 \mathrm{MeV}$ linear electron accelerator and several associated facilities.

$$
\begin{aligned}
& \text { Qualifications - experience with large and complex instrumentation } \\
& \text { - relevant research experience at senior level } \\
& \text { - team leadership and management experience } \\
& \text { - sufficient scientific background to guide research in } \\
& \text { front-line of physics. }
\end{aligned}
$$

A salary will be offered in the range between Dfl. 5000,- and Dfl. 6300,- per month, depending on age and experience.

Enquiries should be addressed to Dr. P.F.A. Goudsmit, Institute for Nuclear Physics Research, P.O. Box 4395, Amsterdam, The Netherlands (telephone 020-930951).

Applications, including a curriculum vitae, list of publications and a least two references should be made before March 15, 1977.

\title{
Physics in Hungary
}

The study of physical problems has always played an important role in the scientific life of Hungary. Outstanding researchers, Loránd Eötvös being a very good example, achieved remarkable results, though mostly in difficult circumstances. Unfortunately, many talented Hungarian physicists, such as Kúrmán, Hevessy, Szilárd, Wigner, and others found it necessary to continue their scientific careers abroad.

Although the roots of the present scientific activities can be traced back to researches carried out in the 1920 s and 1930 s, really rapid development started only after the Second World War. Physics research is carried on in Hungary, as in many other countries, in three types of laboratory: university departments, industrial research institutes and in institutes of the Hungarian Academy of Sciences (HAS). The most

spectacular change has been the evolution of the Academy institutes, as is apparent from the figures listed below:

$$
1950 \quad 1960 \quad 1970
$$

Number of physicists in HAS institutes

Relative allocation per physicist to physics research

While the Academy plays an important role in directing research, the Eötvös Loránd Physical Society is an important social organization enabling physicists from educational centres, research institutes and from industry to exchange ideas in the conferences, schools and in the club-like lecture meetings organized by the Society which is the representative association of Hungarian physicists.

\section{N. Kroó, Budapest}

(Hungarian Academy of Sciences)

\section{Activities in Physics}

Studies in modern theoretical physics started in Hungary in the early thirties. Quantum theory, statistical mechanics and the theory of relativity were the main fields of research. A series of regular high-level seminars with participants from home and abroad helped to promote an interest in these subjects.

After the Second World War the initial task was to lay the foundation of future development by emphasizing the importance of an education in theoretical physics. Simultaneously, a high level school in quantum field theory, that still functions, was established.

The statistical theory of atoms, the study of which was initiated in this early period, is still a subject pursued 
in a few departments of the universities.

Solid state theory also has a traditional place in Hungarian physics research. The advanced level of current theoretical activities is strongly correlated with the overal activities in solid state physics. In the past decade, the main interest of physicists in this field was centred on problems of phase transitions, dilute alloys, magnetic properties of alloys and certain other problems in soild state physics, such as one-dimensional systems and liquid crystals.

An effective connection between theoretical groups and experimental laboratories and with foreign theoretical schools has always provided a substantial driving force in research. As there are more theoretical physicists than members of experimental groups in teaching, theoretical physics is more accessible to the student. Consequently the regeneration of theoretical groups has a more than average chance of taking place.

Experimental studies in nuclear physics started in Debrecen in 1936, but is was not possible to develop them to any great extent until 1955 . To begin with, two HAS institutes were responsible for these studies, the Central Research Institute for Physics, Budapest, founded in 1950 and the Institute for Nuclear Research, Debrecen, founded in 1954. The main particle sources available for nuclear reaction studies are Cockcroft-Walton and Van de Graaff type generators and a $5 \mathrm{MW}$ nuclear reactor. In the past years, nuclear spectroscopy has become a main line of activity using the accelerators of the Joint Institute for Nuclear Research in Dubna. The same equipment is used by a Hungarian group engaged in high energy nuclear interaction studies.

Reactor research was initiated in 1956 in connection with the construction and operation of our research reactor - completed in 1959. Some of the problems of general interest which are being studied are, for example, neutron noise in neutron multiplying systems and the properties of zero power critical assemblies. The experience gained from theoretical and experimental research in reactor physics is a source of help to the reactor engineers in our nuclear power plant programme.

The methods of nuclear physics have had a positive influence on other branches of physics, principally on solid state physics. Structural and dynamical studies by neutron scattering, magnetic and structural studies by the Mössbauer effect, perturbed angular correlation measurements, activation analysis, nuclear chemistry and other techniques appreciably benefit from the methods developed in nuclear physics. Even the solution of biological problems, to which an ever increasing attention has been paid in recent years, is helped by the availability of nuclear methods in our country.

Elementary particle physics has for many years been a subject of intense study all over the world. Our activities in this field began in 1950 with cosmic ray research. When this work started, extended cosmic showers and penetrating showers were investigated by G-M counters, then from 1955 onwards, emulsion techniques were also used. Present studies are centred on the galactic anisotropy of $\approx 10^{11} \mathrm{eV}$ cosmic rays.

As members of the Joint Institute for Nuclear Research, we have taken part since 1956 in high energy physics experiments, using the Dubna $10 \mathrm{GeV}$ synchrophasotron and later the $70 \mathrm{GeV}$ accelerator at Serpukhov. Hungarian participation involves the work of scientists using the accelerator and the evaluation of photographs taken in the accelerator laboratory. Our contribution to the study of weak interactions is perhaps the most important of our results in this cooperation.

Scientific life in theoretical elementary particle physics is lively. Neutrino physics and high energy interactions of elementary particles are some examples where the results are worthy of mention as important contributions to the field.

Spectroscopic studies in Hungary started in 1928 with molecular spectroscopy - which is still the main field of research for some university and other research teams. Nowadays, the main efforts belong to the field of applied research conducted partly in industrial laboratories. Theoretical studies are concerned with the effect of rotational fine structure and of different molecular interactions on optical spectra.

Investigations of the fluorescence of liquids, also a traditional field of study, are proceeding along two main lines, namely the study of the role of luminescence in biological systems, and the investigation of possible laser dyes in order to develop tunable dye lasers.

Laser research originated from the activities in "classical" optics, which were intended to clarify the waveparticle duality problem of photons. Different types of laser were developed and used for research, as well as for a number of practical applications. In recent years the interaction of intense laser light with matter - multiphoton ionization and surface photoeffect has become the centre of interest in laser studies.

Properties of the nonlinear photoeffect and its connection with solid state properties, e.g. plasmons, are being investigated. Linear laser spectroscopy - Raman scattering, Brillouin scattering - and IR and UV methods are used for solid state and molecular studies.

Solid state research in Hungary was connected from the very outset with industrial activity. The products of the Tungsran Works (light bulbs, radio valves, etc.), called for the study of tungsten, glass, fluorescent powders or cathode materials. The study of crystallization and crystal properties of ion crystals has also been a traditional line of research.

At present, two institutes of the HAS and several smaller laboratories are active in the field. Preference on a governmental level is given to this theme because of the potential practical applications. Four main types of material are being studied

a) metals and alloys

b) magnetic materials

c) semiconductors

d) optical materials

Tungsten is still studied with particular regard to the role of dopants. Orderdisorder phenomena in alloys, dilute alloys, decarbonization processes and metal glasses are perhaps the most important problems dealt with in the Hungarian laboratories' contribution to solid state research.

In the fifties, magnetic studies were centred on anisotropy and magnetization processes. Later the investigations were extended to magnetic phase transitions and to the stability of magnetic systems. These problems were the first to require the introduction of modern nuclear methods, such as neutron diffraction and spectroscopy, NMR and Mössbauer techniques which are now widely used.

Advanced semiconductor research started relatively late in our country. The first results concerned instabilities of semiconductor devices. Now the search for new semiconductor materials and the study of processes on the boundary surfaces of these materials are of interest. Nuclear methods like ion implantation for doping and the back-scattering technique for the analysis of impurity profiles have become important tools in semiconductor studies.

Optical materials are used as active or passive elements of lasers or laserbased systems. Laser development and practical applications of lasers are based on these materials. The re- 
search into these materials has evolved from the tradition of growing transparent crystals of optical quality, e.g. artificial quartz, Nal (TI) crystals for elements of infrared spectroscopy, etc. and the resultant study of such crystal/defect structure, etc. It is now stimulated by the demand for such materials in laser research, $\mathrm{LiNb}_{3}, \mathrm{PbMoO}_{4}, \mathrm{TeO}_{2}, \mathrm{KDF}$, etc.

As I have already mentioned, mission-oriented work constitutes a large fraction of solid state research. Very much effort is devoted to the development of memory devices for the computer industry. Magnetic bubble memories are now in their final stage of development. The activities in the field of semiconductors are also notable.

After this brief and by no means complete review of physics research carried out in Hungary, I should like to summarize some general considerations.

1) In the past 25 years, research in physics has grown from the efforts of a few talented but isolated persons to a coordinated, often mission-oriented activity of a large group of scientists with a long-term research strategy.

2) Governmental authorities, responsible for the development of the country's economy, seek support from the scientific community and stimulate scientific activities by financial and other means.

3) Hungary, as a small country, is unable to increase its level of scientific research without intense collaboration with institutes abroad, principally with those in European countries. Multilateral connections, e.g. work in the Joint Institute of Nuclear Research, and bilateral agreements (with almost all European countries) are vital for our scientific progress.

4) The present trend to establish close connections between HAS institutes and industrial laboratories and with university groups is expected to be one of the driving forces of future progress.

It must be borne in mind that in a small country like ours, and at our technological level, it is important that the problems to be studied are selected with the utmost care. We are encouraged to concentrate our efforts upon problems connected with our scientific traditions and occasionally upon tasks which may help to solve problems linked with the national economy.

Finally, we Hungarian physicists would like to have a small share in the development of physics in general for the benefit of people in Europe and all over the world.

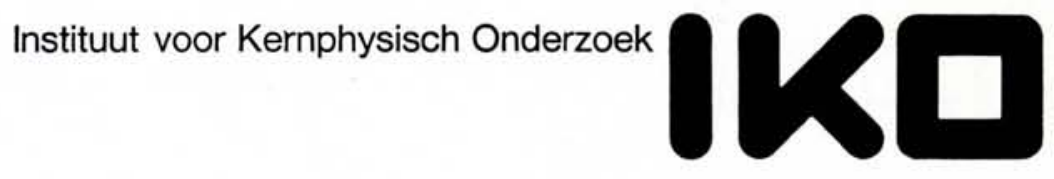

\section{Theoretical Nuclear Physicist}

IKO, the intermediate energy nuclear physics section of the new Dutch national institute for nuclear and high energy physics (NIKHEF) has a (possible tenured) position open for a theoretical nuclear physicist.

The institute is presently constructing a $300 \mathrm{MeV}$ high duty cycle electron linac which will be used for experimental studies in the fields of electron scattering and low energy pion/muon physics.

Applicants are expected to have a good background (PhD and preferably several years of post doctoral experience) in areas of nuclear theory which are closely related to the experimental program.

Applications including resume, references and publication list, should be sent to: Taber de Forest, IKO, Postbus 4395, Amsterdam, the Netherlands (tel. 020-930951) before April 1, 1977.

INTERNATIONAL ADVANCED STUDY INSTITUTE

ON

PATH INTEGRALS AND THEIR APPLICATIONS IN QUANTUM, STATISTICAL AND SOLID STATE PHYSICS

$$
\text { July } 17 \text { - July } 30,1977
$$

University of Antwerpen (Rijksuniversitair Centrum) BELGIUM

Registration by May 15 to :

Scientific Director:

Prof. Dr. J. T. DEVREESE

Leerstoel Toegepaste Wiskunde voor de Wetenschappen

R.U.C.A.

Groenenborgerlaan 171

B-2020 ANTWERPEN
Co-Director :

Prof. Dr. G. J. PAPADOPOULOS

University of Athens

Panepistimiopolis

ATHENS 61 (Greece)

Secretaries :

J. DE SITTER, Antwerpen

L.F. LEMMENS, Antwerpen

\section{Letter to the Editor}

\section{Inventory of Unpublished Documents in 20th Century Physics}

Sir,

The Office for History of Science and Technology at the University of California, Berkeley, is undertaking a world-wide survey of archival holdings related to physics in this century. The published inventory which results will locate and identify correspondence and unpublished papers of approximately one thousand physicists active between 1900 and 1950. Of particular interest is documentation of contact between physicists and intellectuals outside the domain of academic physics.
Readers with special knowledge of :

- Unpublished correspondence with physicists, particularly items in private hands or in archival collections associated primarily with non-physicists ;

- Letters to or from a physicist published in journals or books not likely to be well known to historians of science ;

- Archival holdings of the papers of little-known physicists ;

are urged to relay this information to the Survey of Archives,

c/o Office for History of Science and Technology 470 Stephens Hall University of California Berkeley, California 94720.

B.R. Wheaton 